\title{
From probabilistic forecasts to statistical scenarios of short-term wind power production
}

Pinson, Pierre; Papaefthymiou, George; Klockl, Bernd; Nielsen, Henrik Aalborg; Madsen, Henrik

Published in:
Wind Energy

Link to article, DOI:

$10.1002 /$ we.284

Publication date:

2009

Document Version

Early version, also known as pre-print

Link back to DTU Orbit

Citation (APA):

Pinson, P., Papaefthymiou, G., Klockl, B., Nielsen, H. A., \& Madsen, H. (2009). From probabilistic forecasts to statistical scenarios of short-term wind power production. Wind Energy, 12(1), 51-62.

https://doi.org/10.1002/we.284

\section{General rights}

Copyright and moral rights for the publications made accessible in the public portal are retained by the authors and/or other copyright owners and it is a condition of accessing publications that users recognise and abide by the legal requirements associated with these rights.

- Users may download and print one copy of any publication from the public portal for the purpose of private study or research.

- You may not further distribute the material or use it for any profit-making activity or commercial gain

- You may freely distribute the URL identifying the publication in the public portal 


\title{
From Probabilistic Forecasts to Statistical Scenarios of Short-term Wind Power Production
}

\author{
Pierre Pinson*, Henrik Madsen
}

DTU Informatics, Technical University of Denmark, Lyngby, Denmark

Henrik Aa. Nielsen

ENFOR A/S, Hørsholm, Denmark

DTU Informatics, Technical University of Denmark, Lyngby, Denmark

George Papaefthymiou

Power Systems and Markets, Ecofys Germany GmbH, Berlin, Germany

Electrical Power Systems Laboratory, TU Delft, Delft, the Netherlands

Bernd Klöckl

Verbund Austrian Power Grid (APG), Vienna, Austria

\begin{abstract}
Short-term (up to 2-3 days ahead) probabilistic forecasts of wind power provide forecast users with a highly valuable information on the uncertainty of expected wind generation. Whatever the type of these probabilistic forecasts, they are produced on a per horizon basis, and hence do not inform on the development of the forecast uncertainty through forecast series. However, this additional information may be paramount for a large class of time-dependent and multi-stage decision-making problems e.g. optimal operation of combined wind-storage systems or multiple-market trading with different gate closures. This issue is addressed here by describing a method that permits the generation of statistical scenarios of short-term wind generation that accounts for both the interdependence structure of prediction errors and the predictive distributions of wind power production. The method is based on the conversion of series of prediction errors to a multivariate Gaussian random variable, the interdependence structure of which can then be summarized by a unique covariance matrix. Such matrix is recursively estimated in order to accommodate long-term variations in the prediction error characteristics. The quality and interest of the methodology are demonstrated with an application to the test case of a multi-MW wind farm over a period of more than two years.
\end{abstract}

Keywords: wind power, forecasting, uncertainty, multivariate Gaussian random variable, scenarios.

\footnotetext{
* Corresponding author:

P. Pinson, DTU Informatics, Technical University of Denmark,

Richard Petersens Plads (bg. 321 - 020), DK-2800 Kgs. Lyngby, Denmark.

Tel: +45 4525 3428, fax: +45 4588 2673, email: pp@imm.dtu.dk, webpage: www.imm.dtu.dk/ pp
} 


\section{Introduction}

Increasing the value of wind generation through the improvement of prediction systems' performance is one of the priorities in wind energy research needs for the coming years [1]. Today, most of the existing wind power prediction methods provide end-users with point forecasts [2]. The parameters of the models involved are commonly obtained with minimum least square estimation. If denoting by $p_{t+k}$ the measured power value at time $t+k, p_{t+k}$ can actually be seen as a realization of a random variable $P_{t+k}$ which summarizes the potential power production values at that time. In parallel, write $\hat{p}_{t+k \mid t}$ a point forecast issued at time $t$ for lead time $t+k$, based on a given model, its parameters, and the available information on the process up to time $t$ (i.e. including various measurements and meteorological forecasts). The point forecast $\hat{p}_{t+k \mid t}$ then corresponds to the conditional expectation of $P_{t+k}$, which is a single summary statistics for this random variable.

Owing to their highly variable level of accuracy, a large part of the recent research works has focused on associating point forecasts with appropriate information on their situation-specific uncertainty. Such information may take the form of risk indices or probabilistic forecasts [3]. The latter ones are the most common and utilized in practice today, even though the use of prediction risk indices also comprises a promising alternative (or complementary) approach [4]. Probabilistic predictions can be either derived from meteorological ensembles [5], based on physical considerations [6], or finally produced from one of the numerous statistical methods that have appeared in the literature, see $[4,7,8,9,10]$ among others. Their aim is to give more information on the random variable $P_{t+k}$ than the simple summary statistics given by a point forecast. If appropriately incorporated in decision-making methods, they permit to significantly increase the value of wind generation. Recent developments in that direction concentrate on e.g. dynamic reserve quantification [11], optimal operation of combined wind-hydro power plants [12,13] or on the design of optimal trading strategies [14].

Probabilistic forecasts are generated on a per look-ahead time basis. They do not inform on the development of the prediction errors through the prediction series related to a given prediction time point ( $t$ above), since they neglect the interdependence structure of forecast errors among lookahead times. However, this interdependence structure is of particular importance for many timedependent and multi-stage decision-making processes e.g. the economic operation of conventional generation in combination to wind power output, the optimal operation of wind-storage systems, or alternatively the design of trading strategies in a multi-market environment with different gate closures. In order to satisfy this additional requirement, it is proposed here to generate scenarios of short-term wind power production. They should respect the probabilistic forecasts for the next period, and additionally rely on the most recent information about the interdependence structure of the prediction errors. Such kind of scenarios are indeed required by new methodologies specially designed either for optimal integration of stochastic generation into energy systems [15] or for optimal planning in presence of distributed storage devices [16]. The main objective of the present paper is to introduce the general framework for the derivation of such scenarios of short-term wind power production. This framework may be extended in the future in order to also integrate spatial and spatio-temporal aspects of forecast uncertainty, which are relevant for e.g. congestion management or probabilistic load flows. In addition, the method presented has some generic value since any type of probabilistic forecasts may be used as input. As it is often the case today for advanced forecasting methodologies, a requirement though is that measurements of wind power production for the site considered are regularly made available, preferably in an online setting. An example application for the dynamic sizing of storage requirements that would permit to face regulation costs in an electricity market environment, and based on the scenarios introduced in the present paper, is described in [17].

Nonparametric probabilistic forecasts of wind generation are introduced in a first Section, and a brief overview of their required and desirable properties is given. If nonparametric forecasts are 
considered here as input to the method, it is because they consist of the most general form of probabilistic predictions (since they also encompass parametric ones). The method for generating statistical scenarios of wind generation from nonparametric probabilistic forecasts is then described. It is based on the transformation of the set of random variables composing probabilistic forecast series into a single multivariate Gaussian variable. For such a multivariate Gaussian variable, the complete interdependence structure of its elements can be summarized by its covariance matrix only. In order to accommodate the nonstationary characteristics of the wind power forecast uncertainty (cf. discussion in [10]), this covariance matrix is tracked with a recursive estimation method. Results from the application of the described method are given subsequently. The test case considered is that of a multi-MW wind farm located in the North of Denmark, for which point forecasts, probabilistic forecasts and related power measurements are available over a period of more than two years. Emphasis is on discussing the Gaussian assumption on the transformed random variables, on the evaluation of the probabilistic correctness of the generated scenarios, and finally on the time-varying interdependence structure of prediction errors. Concluding remarks end the paper with perspectives on applications and future developments.

\section{Nonparametric probabilistic forecasts of wind power}

Even though nonparametric probabilistic predictions may take the form of quantile, interval or density forecasts, the basic quantity to be considered is the quantile forecast, since the other two may be expressed in terms of two or more quantile forecasts. Write $f_{t+k}$ the probability density function of $P_{t+k}$, and let $F_{t+k}$ be the related cumulative distribution function. Provided that $F_{t+k}$ is a strictly increasing function, the quantile $q_{t+k}^{(\alpha)}$ with proportion $\alpha \in[0,1]$ of the random variable $P_{t+k}$ is uniquely defined as the value $x$ such that

$$
\mathrm{P}\left(P_{t+k}<x\right)=\alpha, \text { or } q_{t+k}^{(\alpha)}=F_{t+k}^{-1}(\alpha)
$$

A quantile forecast $\hat{q}_{t+k \mid t}^{(\alpha)}$ with nominal proportion $\alpha$ is an estimate of $q_{t+k}^{(\alpha)}$ produced at time $t$ for lead time $t+k$, given the information set $\Omega_{t}$ at time $t$.

For most decision-making processes, such as power system operation, a single quantile forecast is not sufficient for making an optimal decision for a given lead time. It is instead necessary to have the whole information about the random variable $P_{t+k}$ for each horizon over a period ranging from few hours to several days ahead (see e.g. [14]). If no assumption is made about the shape of the target distributions, a nonparametric forecast $\hat{f}_{t+k \mid t}$ of the density function of the variable of interest (i.e. wind power production) at lead time $t+k$ can be produced by gathering a set of $m$ quantile forecasts

$$
\hat{f}_{t+k \mid t}=\left\{\hat{q}_{t+k \mid t}^{\left(\alpha_{i}\right)} \mid 0 \leq \alpha_{1}<\ldots<\alpha_{i}<\ldots<\alpha_{m} \leq 1\right\}
$$

that is, with chosen nominal proportions spread on the unit interval. These types of probabilistic forecasts are hereafter referred to as predictive distributions. $\hat{F}_{t+k \mid t}$ denotes the cumulative distribution function related to $\hat{f}_{t+k \mid t}$. Note that interval forecasts correspond to the specific case for which only two quantiles are quoted, and whose nominal proportions are chosen to be symmetric around the median. In addition, the family of nonparametric probabilistic forecasts described by a set of quantiles as in equation (2) also encompasses that of parametric ones, as any type of probabilistic distributions can be summarized by a sufficient number of its quantiles.

A requirement for nonparametric probabilistic forecasts is that the nominal probabilities, i.e. the nominal proportions of quantile forecasts, are respected in practice. Over an evaluation set of significant size, the empirical (observed) and nominal probabilities should be as close as possible. Asymptotically, this empirical coverage should exactly equal the pre-assigned probability. That 
required property is commonly referred to as reliability. Besides this requirement, it is highly desirable that probabilistic predictions provide forecast users with a situation-dependent assessment of the prediction uncertainty. The shape of predictive distributions should then vary depending on various external conditions. For the example of wind power forecasting, it is intuitively expected that prediction intervals (for a given nominal coverage rate) should not have the same size when predicted wind speed equals zero and when it is near cut-off speed. This desirable property of probabilistic forecasts is commonly referred to as their sharpness of resolution. For a more thorough discussion on these various aspects, see [18, 19].

\section{Generating scenarios of wind power production}

Given the nonparametric probabilistic forecasts described above, it is necessary to capture the interdependence structure of the prediction errors among the set of forecast horizons. For that purpose, it is proposed here to take advantage of a fundamental property of reliable probabilistic predictions, which is such that prediction errors can be made Gaussian by applying a suitable transformation. The interdependence structure of the transformed prediction errors can then be summarized by a unique covariance matrix. Long-term variations in the interdependence structure of prediction errors are tracked by recursively estimating this covariance matrix with an exponential forgetting scheme. The main points of the method are described in the following.

\section{The Gaussian multivariate random variable}

Let us focus on a single look-ahead time $k$. As explained above, the series of predictive distributions $\left\{\hat{f}_{t+k \mid t}\right\}_{t}$ for that look-ahead time are defined as reliable if the observed proportions for each of the quantiles correspond to the nominal ones [18]. In such a case, the random variable $Y_{k}$ whose realization $Y_{k}^{(t)}$ at time $t$ is defined by

$$
Y_{k}^{(t)}=\hat{F}_{t+k \mid t}\left(p_{t+k}\right), \quad \forall t
$$

is distributed uniform on the unit interval, i.e. $Y_{k} \sim \mathrm{U}[0,1]$. Consider for instance predictive distributions defined by quantiles whose nominal proportions are uniformly spread between 0 and 1 . This property translates to saying that there is the same probability the measured power value at time $t+k$ falls in any of the interval defined by two neighboring quantile forecasts $\hat{q}_{t+k \mid t}^{\left(\alpha_{i}\right)}$ and $\hat{q}_{t+k \mid t}^{\left(\alpha_{i+1}\right)}$, $i=1, \ldots, m-1$. Note that in practice, the continuous cumulative distribution function to be used in the above equation is obtained by fitting a smooth curve through the set of $m$ quantile forecasts, cf. equation (2).

Then, given the uniformly distributed random variable $Y_{k}$, a straightforward way to obtain a Normally distributed variable $X_{k}$ is to apply a second transformation, that uses the probit function, which corresponds to the inverse of the Gaussian cumulative distribution function. The probit function is defined as

$$
\Phi^{-1}: p \rightarrow \sqrt{2} \operatorname{erf}^{-1}(2 p-1)
$$

where ' $\mathrm{erf}^{-1}$ ' is the inverse error function. Consequently, the transformation of $Y_{k}$ to $X_{k}$ is obtained by applying the probit function to every realization $Y_{k}^{(t)}$, i.e.

$$
X_{k}^{(t)}=\Phi^{-1}\left(Y_{k}^{(t)}\right), \quad \forall t
$$

such that the random variable $X_{k}$ is distributed Gaussian with zero mean and unit standard deviation, $X_{k} \sim \mathcal{N}(0,1)$. 
Considering the transformed random variables $X_{k}$ for each look ahead time, it is assumed that the random vector $\mathbf{X}=\left(X_{1}, X_{2}, \ldots, X_{K}\right)^{\top}$, where $K$ is the maximum forecast horizon, follows a multivariate Gaussian distribution, $\mathbf{X} \sim \mathcal{N}\left(\mu_{0}, \Sigma\right)$, with the vector $\mu_{0}$ of mean values being a vector of zeros. In addition, $\Sigma$ is the covariance matrix that contains the whole information about variance-covariance of the random variables $X_{k}, k=1, \ldots, K$. It has 1 -values on its diagonal, since the diagonal elements give the variance of each of the random variables.

The assumption of $\mathbf{X}$ being a multivariate Gaussian distribution is the simplest assumption one can make about the interdependence structure of the individual random variables $X_{k}$. More complex interdependence structures could be modeled by using the copula theory, which involves the modeling of the rank correlation of the $Y_{k}$-variables [15, 20]. This would not affect the other parts of the proposed method.

\section{Recursive estimation of the covariance matrix}

In the context of wind power forecasting applications, measurements are regularly collected and subsequently used for updating the parameters of the physical/statistical models used as a basis for prediction. A similar approach based on a recursive formulation can be applied for the adaptive estimation of $\Sigma$.

Write $\Sigma_{t}$ the covariance matrix estimated from observations $\mathbf{X}^{(j)}$ of the random variable $\mathbf{X}$ random variable up to time $t$ (thus implying $j=1, \ldots, t$ ). An unbiased estimate of $\Sigma_{t}$ is commonly given by

$$
\Sigma_{t}=\frac{1}{t-1} \sum_{j=1}^{t} \mathbf{X}^{(j)} \mathbf{X}^{(j)}{ }^{\top}
$$

with.$^{\top}$ the transposition operator.

Given that the covariance estimation is based on a normalized sum, a recursive formulation for the updating of $\Sigma_{t-1}$ when new observations are made available at time $t$ is readily obtained as

$$
\Sigma_{t}=\left(\frac{t-2}{t-1}\right) \Sigma_{t-1}+\left(\frac{1}{t-1}\right) \mathbf{X}^{(t)} \mathbf{X}^{(t)} \top^{\top}
$$

Considering that the process characteristics are slowly varying leads to the application of an exponential forgetting scheme to the recursive updating formula in equation (7). This would yield

$$
\Sigma_{t}=\lambda\left(\frac{t-2}{t-1}\right) \Sigma_{t-1}+\left(1+\lambda\left(\frac{1}{t-1}-1\right)\right) \mathbf{X}^{(t)} \mathbf{X}^{(t)}{ }^{\top}
$$

where $\lambda$ is the forgetting factor, $\lambda \in[0,1)$. This updating formula is such that when $t$ tends towards infinity, it becomes

$$
\Sigma_{t}=\lambda \Sigma_{t-1}+(1-\lambda) \mathbf{X}^{(t)} \mathbf{X}^{(t)}{ }^{\top}
$$

which corresponds to the classical formula for exponential forgetting, see e.g. [21]. The covariance matrix is initialized by setting all its off-diagonal elements to 0 and its diagonal elements to 1 .

Owing to potential small deviations from perfect reliability of the probabilistic forecasts of wind generation used as input, it may occur that $\Sigma_{t}$ slightly deviates from what would be a suitable covariance matrix of a unit multivariate Normal variable. By assuming that such deviation is only an issue related to variance scaling, applying an appropriate transformation at each time step 
permits to recalibrate $\Sigma_{t}$. It is defined as

$$
\Sigma_{t}=\Sigma_{t} \oslash\left(\sigma_{t} \sigma_{t}^{\top}\right)
$$

with $\sigma_{t}$ the vector of standard deviations i.e. corresponding to the square root of the diagonal elements of $\Sigma_{t}$, and where $\oslash$ denotes the element-by-element division. This transformation is equivalent to normalizing past realizations of $\mathbf{X}$ by $\sigma_{t}$ so that each component $X_{k}$ follows a unit Normal distribution.

\section{Scenario generation}

At time $t$ a set of predictive distributions $\hat{f}_{t+k \mid t}$ for each look-ahead time $k, k=1, \ldots, K$, is available. In parallel, the most up-to-date estimate of the covariance matrix is $\Sigma_{t-K}$, since the most recent forecast series for which all forecast values can be compared with measurements is that generated at time $t-K$. From these two pieces of information, the procedure for obtaining a number $d$ of scenarios of wind power production for the following $K$ look-ahead times is as following:

- (i) one uses a multivariate Gaussian random number generator with zero mean and covariance matrix $\Sigma_{t-K}$ in order to have $d$ realizations of the random variable $\mathbf{X}, \mathbf{X} \sim \mathcal{N}\left(\mu_{0}, \Sigma_{t-K}\right)$. Denote by $\mathbf{X}^{(i)}$ the $i^{\text {th }}$ of these $d$ realizations;

- (ii) for each horizon $k, d$ realizations $Y_{k}^{(i)}$ of the uniform variable $Y_{k}$ are obtained by applying the inverse probit function $\Phi$ to each component of $\mathbf{X}^{(i)}$,

$$
Y_{k}^{(i)}=\Phi\left(X_{k}^{(i)}\right), \quad \forall k, i
$$

- (iii) the scenarios of wind power production finally result from the application, for each lookahead time $k$, of the inverse cumulative distribution function $\hat{F}_{t+k \mid t}^{-1}$ to the $d$ realizations $Y_{k}^{(i)}$ of $Y_{k}$ for that look-ahead time. If having $\hat{p}_{t+k \mid t}^{(i)}$ the power value at lead time $t+k$ for the $i^{\text {th }}$ scenario, this writes

$$
\hat{p}_{t+k \mid t}^{(i)}=\hat{F}_{t+k \mid t}^{-1}\left(Y_{k}^{(i)}\right), \quad \forall k, i
$$

By applying these three steps, one obtains $d$ scenarios of short-term wind power production for the following $K$ look-ahead times. They can then be used as input to decision-making methods or power system models (in a Monte Carlo simulation framework for instance) in order to evaluate the potential impact of wind power forecast uncertainty and interdependence of forecast errors on decisions made from point forecasts only. Ideally, future decision-making methods and power system models would be developed following a probabilistic framework, permitting to define different optimal decisions depending on the forecast-user's sensitivity to consequences of forecast errors.

\section{Application results}

In this Section, focus is given to the application of the method for the generation of scenarios of short-term wind power production introduced above to a real-world test case, in order to demonstrate the quality of this method. After describing the case-study considered, it will be verified that transformed variables are indeed close to being Gaussian, and that generated scenarios are probabilistically correct over the evaluation period, i.e. that they respect the probabilistic forecasts used as input. Finally, the evolution of the interdependence structure of forecast errors through time is discussed. 


\section{Description of the case-study}

Predictions are produced for a Danish onshore wind farm with a nominal capacity $P_{n}$ of 21MW. The period for which both predictions and related power measurements are available goes from March 2001 until end of April 2003. The point predictions result from the application of the Wind Power Prediction Tool (WPPT) method [22], which uses meteorological predictions of wind speed and direction as input, as well as historical measurements of power production. Point predictions have an hourly resolution (similarly to measurements) up to 43-hour ahead. They are updated every hour. All predictions and measurements are normalized by the nominal capacity of the wind farm and hence expressed in percentage of $P_{n}$. The dataset consists of 16900 point prediction series.

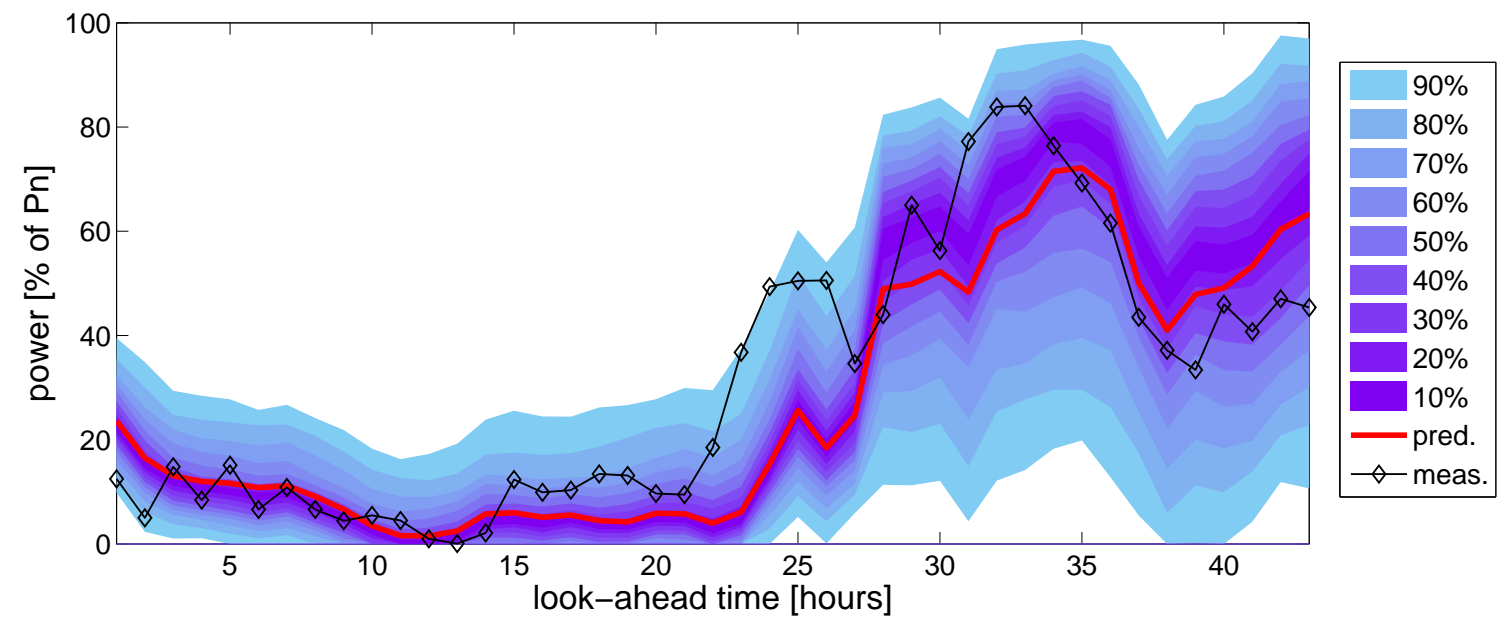

FIGURE 1: Example of probabilistic predictions of wind generation in the form of nonparametric predictive distributions. Point predictions are obtained from wind forecasts and historical measurements of power production, with the WPPT method. They are then accompanied with interval forecasts produced with adaptive quantile regression. The nominal coverage rates of the prediction intervals are set to 10, 20, .., and $90 \%$.

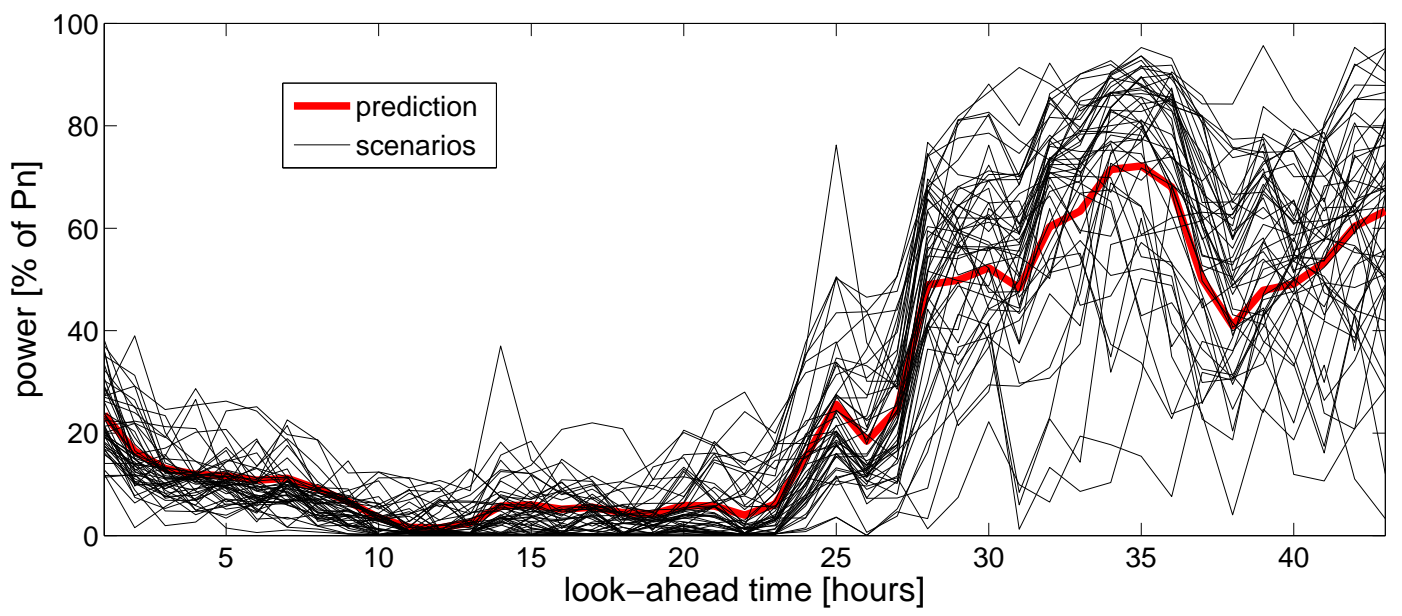

FIGURE 2: Example of wind power point predictions with 50 alternative scenarios produced from the method described in the paper (for the same period as in Fig. 1). The point prediction series correspond to the conditional expectation of wind generation for each look-ahead time. The associated scenarios reflect the prediction uncertainty and the interdependence structure of predictions errors.

Nonparametric probabilistic forecasts are produced with adaptive quantile regression [10]. Predictive distributions are given by 19 quantile forecasts whose nominal proportions range from 0.05 to 0.95 by 0.05 increments. From a probabilistic point of view, since it is not possible to exclude any 
possibility, the predictive quantiles with nominal proportions 0 and 1 are always set to normalized power values of 0 and 1 , respectively, whatever the look-ahead time. The very tails of predictive distributions are parameterized with exponential tails, thus reflecting the unlikeliness of extreme events. The quality of probabilistic forecasts produced with this method and for this particular case-study is evaluated and discussed in [18], while additional evaluation results may be found in [10]. Adaptive quantile regression has been shown to have an acceptable level of reliability, and a superior overall skill when compared with other nonparametric probabilistic forecasting methods of the state of the art.

Fig. 1 gives an example of such probabilistic forecasts of wind generation, in the form of a fan chart. In parallel, Fig. 2 depicts a set of 50 scenarios of power production for the same period, generated from the method introduced, along with the traditionally provided point forecasts for the coming period. For this illustration only 50 scenarios are produced, but since the method is not highly computationally expensive, one can raise this number to several thousands in order to be directly used in e.g. Monte-Carlo simulations for decision-making. The output of such simulations would for instance consist of a distribution of potential unwanted costs of making a decision based on the provided point forecasts, owing to the related prediction errors. For the following evaluation, the number of scenarios is set to 10000 . The second parameter of the method is the forgetting factor $\lambda$ that permits an adaptive tracking of the interdependence structure of prediction errors. It is set here to 0.995 , which corresponds to an effective number of 200 observations (approximately one week). The choice for this value may be seen as quite arbitrary, though coherent with the time scale of motion of large meteorological systems over Europe. To our knowledge, there is no theoretical background so far that would allow to define analytically or numerically an optimal forgetting factor when tracking the type of covariance matrices considered in the present paper. This will be the topic of further research work.

\section{Gaussian assumption on transformed random variable}

The assumption such that the transformed random variable $X_{k}$ is Gaussian is discussed here. For that purpose, the realizations $X_{k}^{(t)}$ of $X_{k}$ are collected over the whole dataset. In principle, one would use a test of Gaussianity for verifying that such an assumption is acceptable. Actually, this kind of test could also be used for verifying the reliability of nonparametric probabilistic forecasts. For literature on Gaussianity testing, we refer to [23] and references therein. However, it is not expected that probabilistic forecasts are perfectly reliable in an hypothesis testing framework, as discussed in [18]. This is owing to the autocorrelation pattern of series of prediction errors (in turn imposed by the inertia of uncertainty in the prediction of meteorological phenomena), which would invalidate the significance level of relevant tests. Here, in order to illustrate and discuss the Gaussianity of the transformed random variables, their distributions are compared to the probability distribution function of a $\mathcal{N}(0,1)$ distribution. This is done both visually and by comparing moments of the various distributions. Results are given and discussed here for the example of the 18-hour ahead forecast horizon only $(k=18)$, due to the fact that those for other look-ahead times are qualitatively similar.

Fig. 3 gives the example of the distribution of $X_{k}$ realizations (for the specific case of $k=18$ ), along with a plot of the probability distribution function of a $\mathcal{N}(0,1)$. The empirical distribution of observations appears to be slightly left-skewed and sharper than the probability distribution function of a $\mathcal{N}(0,1)$, though they look very similar.

In parallel, the four moments of the empirical distributions of $X_{k}$ realizations $(k=1, \ldots, 43)$, i.e. their mean $\mu_{X}(k)$, their standard deviation $\sigma_{X}(k)$, their skewness $\gamma_{X}(k)$ and finally their excess kurtosis $\kappa_{X}(k)$, are calculated. Their average value over the set of look-ahead times and related standard deviation, denoted by ${ }^{-}$and $\sigma($.$) respectively, are gathered in Table 1$. 


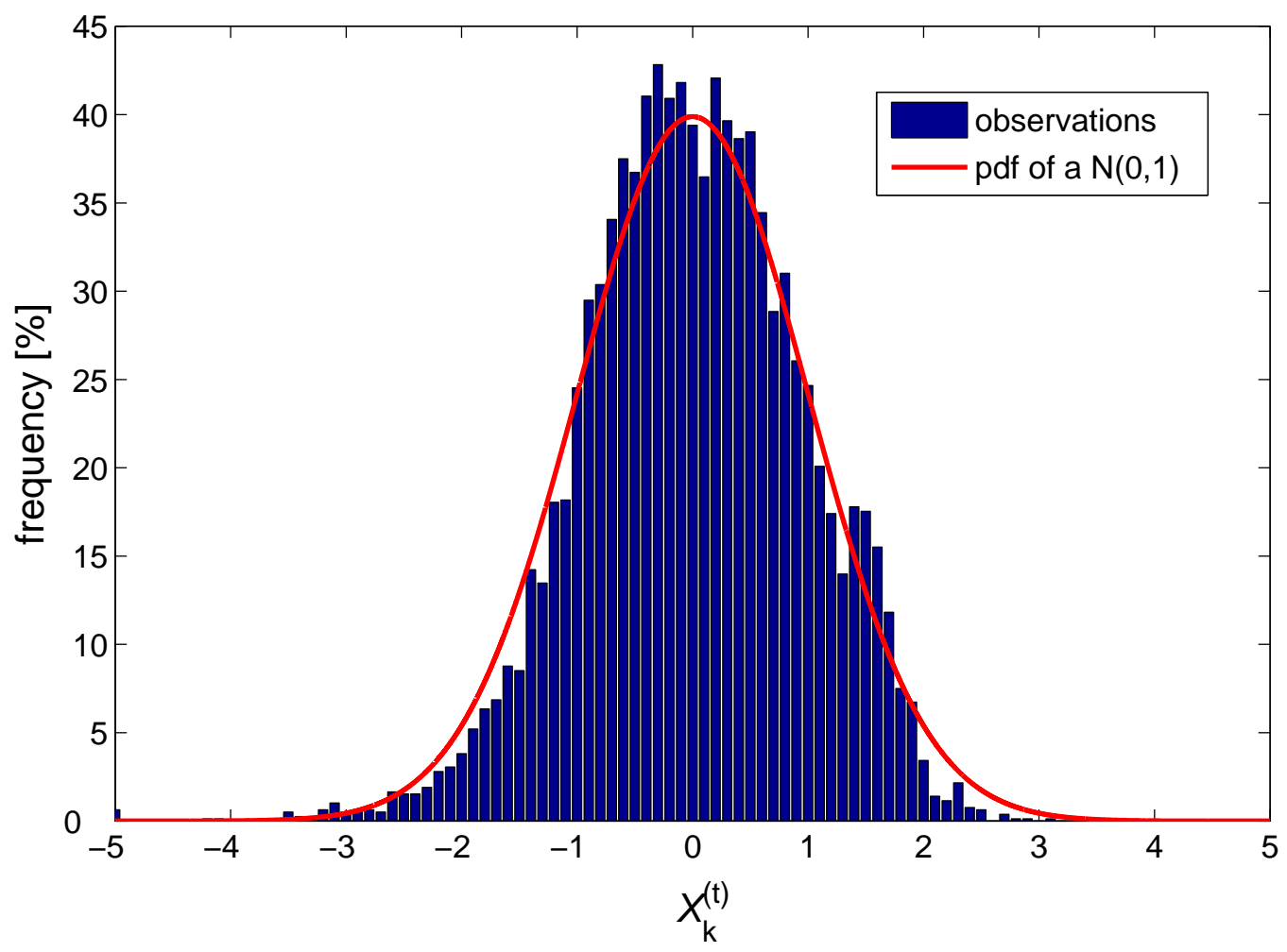

FIGURE 3: Visual comparison between the set of observations $\left\{X_{k}^{(t)}\right\}$ (for $k=18$, i.e. for the transformed random variable related to 18-hour ahead forecasts) and the probability distribution function (pdf) of a $\mathcal{N}(0,1)$ distribution.

TABLE 1: Summary statistics related to the moments of the distributions of the observations of the transformed random variables $X_{k}$.

\begin{tabular}{cccc}
\hline \hline $\bar{\mu}_{X}$ & $\bar{\sigma}_{X}$ & $\bar{\gamma}_{X}$ & $\bar{\kappa}_{X}$ \\
0.0018 & 0.95 & -0.20 & 0.36 \\
\hline$\sigma\left(\mu_{X}\right)$ & $\sigma\left(\sigma_{X}\right)$ & $\sigma\left(\gamma_{X}\right)$ & $\sigma\left(\kappa_{X}\right)$ \\
0.0015 & 0.01 & 0.05 & 0.24 \\
\hline \hline
\end{tabular}

Whatever $k$, the distributions are slightly left-skewed, as it is observed for the example of $k=18$ in Fig. 3. In addition, the excess kurtosis is positive, with low values though, indicating that $X_{k^{-}}$ observations distributions are sharper in their central part, and have longer tails, than a $\mathcal{N}(0,1)$. These slight deviations from a perfect Gaussian shape are due to the fact that the nonparametric probabilistic forecasts are not perfectly reliable in the low power range [18]. Regarding the first two moments, one sees that transformed variables are clearly centred with a standard deviation close to 1 . Globally, even if it was commented that empirical distributions may not be deemed as Gaussian in a hypothesis framework, both visual comparison and evaluation of the distribution moments shows that such distributions are close to being Gaussian. 


\section{Probabilistic correctness of generated scenarios}

In a second stage, it is verified if the generated scenarios are probabilistically correct, i.e. if they respect the marginal distributions of the probabilistic forecasts used as input. This evaluation is based on the required property such that the proportion of generated scenarios that lies between two successive quantiles of a given predictive distribution should correspond to the difference in nominal probability between these two quantiles. For instance, for a given predictive distribution, $5 \%$ of generated scenarios should lie in the interval formed by the quantiles with nominal proportions 0.4 and 0.45 . Such an evaluation can be summarized in a Probability Integral Transform (PIT) histogram, as introduced in e.g. [19]. The PIT histogram of Fig. 4 summarizes this evaluation over the whole dataset and for all horizons, since it has been witnessed that the probabilistic correctness was not significantly different for the various look-ahead times.

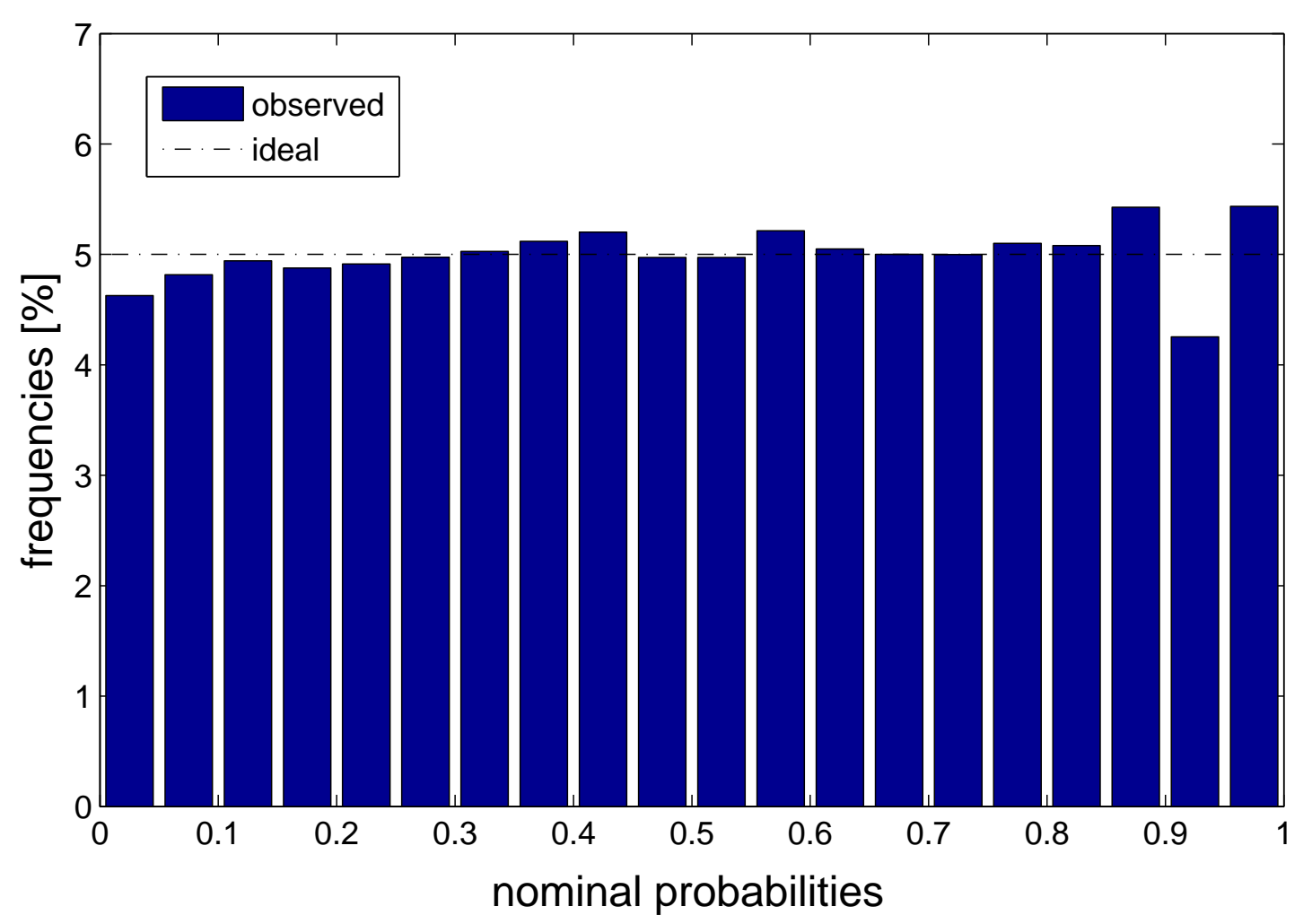

FIGURE 4: Evaluation of the probabilistic correctness of generated scenarios with a PIT histogram. The ideal case of perfect probabilistic correctness is represented by the dash-dot line. This PIT histogram gathers the results for all look-ahead times.

The ideal situation corresponds to the case for which each bin would contain $5 \%$ of the scenarios. The deviations from this ideal situation are low here, indicating that the generated scenarios have distributions similar to that of the input predictive distributions, and this all over the range of nominal probabilities. Note that such evaluation only shows that the generated scenarios are probabilistically correct in the sense that they respect the marginal distributions used as input. If these marginal distributions used as input were not reliable, the generated scenarios would also not be reliable. In addition, non-reliability of the input marginal distributions would also translate to transformed variables $X_{k}$ significantly deviating from Gaussian, and thus disabling the applicability of the method proposed in the present paper. The reliability of scenarios of short-term wind generation being verified, their resolution and added value in decision-making processes or power 
system models will be directly driven by the resolution of input predictive distributions, as well as by the capacity of the covariance matrix of the $\mathrm{X}$ random variable to represent changes in the interdependence structure of prediction errors.

\section{On the tracking of the interdependence structure of forecast errors}

The interdependence structure of forecast errors (at time $t$ ) for the various look-ahead times is summarized by $\Sigma_{t}$ corresponding to the most recent update of the covariance matrix of the multivariate Gaussian variable X (cf. equation (9)). Therefore, in order to follow the evolution of this interdependence structure, on has to track changes in $\Sigma_{t}$ as $t$ increases through the dataset. Figure 5 proposes a visualization of the elements of $\Sigma_{t}$ for two points in time of the dataset considered, i.e. $t=300$ hours and $t=2500$ hours. The $x$ - and $y$-axis are for the prediction horizon $k$. Each pixel gives the covariance between two forecast horizons, and hence variance values for each forecast horizon on the diagonal. The colour scale goes from white for the largest values to black for the lowest ones. In both plots, values of the elements of $\Sigma_{t}$ are contained within the range [-0.2,1].
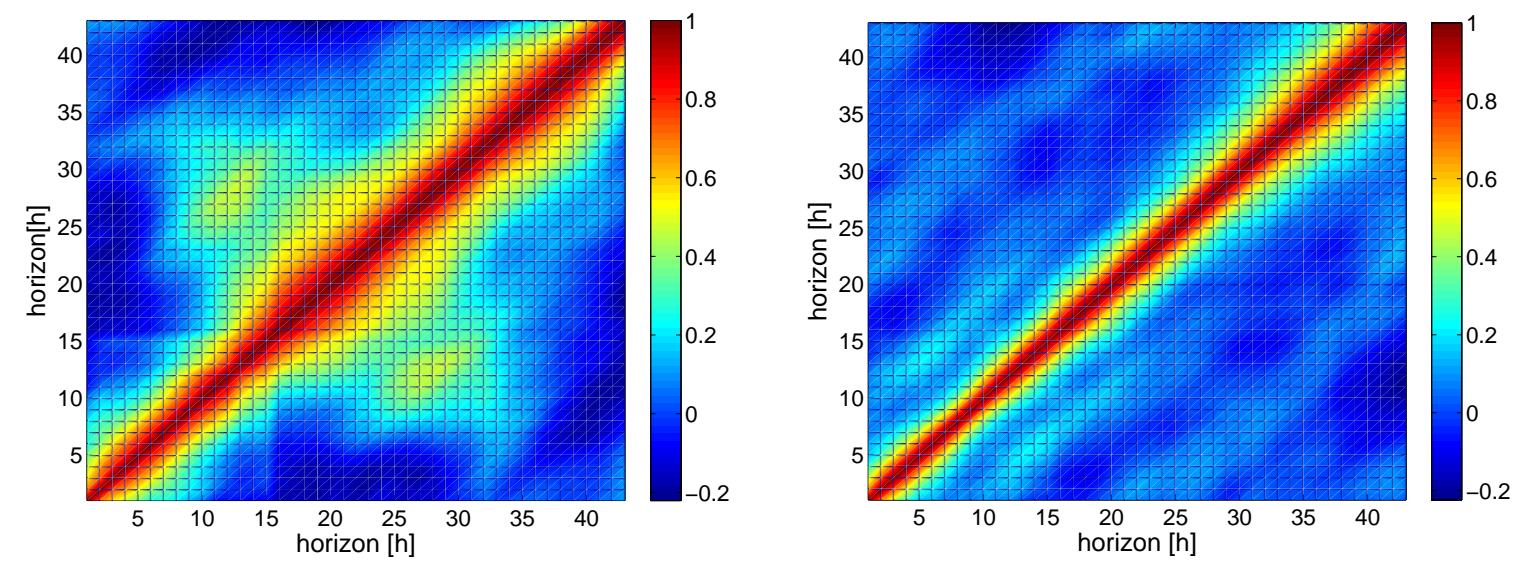

FIGURE 5: Visualization of the covariance matrices of the multivariate normal random variable that permits to estimate and track the interdependence structure of prediction errors. Plots are for two time points of the dataset: after 300 hours (left) and after 2500 hours (right).

The maximum values of the elements of $\Sigma_{t}$ obviously lie on the diagonal, as it has been explained above that the variance of transformed prediction errors for each prediction horizon would be very close to 1 . Covariance values sharply decrease as the difference between prediction horizons increases. However, one may notice that covariance patterns differ for the two points in time. In the first case ( $t=300$ hours), there is a significantly stronger covariance pattern, with more specifically a high correlation between transformed prediction errors for horizons between 12 and 32-hour ahead. Such phenomenon may relate to the point prediction method (or more precisely to the meteorological predictions used as input) not capturing a diurnal component of the wind generation at this site. This covariance pattern is not present in the second case, i.e. for $t=2500$ hours. Such change in the covariance pattern captured by the covariance matrix $\Sigma_{t}$ illustrates the interest of its adaptive tracking. Note that the interdependence structure of forecasts errors is slowly varying with time, but it may also be influenced by some explanatory variables e.g. wind direction. 


\section{Conclusions and Perspectives}

Probabilistic forecasts of wind generation are a highly valuable input to a number of decisionmaking problems related the management or trading of wind generation. However, the fact that they do not provide any information on the interdependence structure of prediction errors make them difficult to interpret for a large class of operational tasks, for which successive decisions are interdependent. This concerns for instance the optimal operation of wind in combination with conventional generation (or storage), or the design of trading strategies for the participation in multiple markets with different gate closures. Indeed for such problems, the appropriate input concerning short-term wind power production must consist of production scenarios that provide the whole information about the development of forecast uncertainty through the coming period. In the present paper, we have described a method that permits to generate scenarios of wind generation from probabilistic forecasts of wind power. It has been shown that such scenarios respect both the predictive distributions and the interdependence structure of prediction errors.

The various qualities of the methodology have been illustrated on the test case of a multi-MW wind farm, for which both point predictions and nonparametric probabilistic forecasts were obtained from state-of-the-art methods i.e. WPPT and adaptive quantile regression, respectively. Future developments related to the method for generating scenarios will focus on the covariance matrix of the transformed random variable. More specifically, it is envisaged in a first stage to propose a method for an optimal choice of the forgetting factor, since the level of time-adaptive ability of the method may be crucial for online applications. Then, the possibility of making the covariance matrix a function of explanatory variables (e.g. level of predicted power of indicative variables on the meteorological situations) in order to account for their impact on the interdependence structure of prediction errors will be studied. Mainly, it is expected that prevailing meteorological situations have a high influence of the interdependence structure of prediction errors. For those that are less easily predictable, and which would lead to phase errors for instance, forecast errors will be significantly more interdependent. Finally, the method will be generalized in order to account for the spatio-temporal aspects of this interdependence structure, or to couple this interdependence structure with that of other relevant variables for decision making e.g. load, solar power generation or market prices.

Broader perspectives relate to the use of wind generation scenarios in a range of decision-making problems, and to the verification of the resulting benefits.

\section{Acknowledgments}

The authors greatly acknowledge Vattenfall Denmark for providing both meteorological forecasts and power data used in the present paper. In addition, acknowledgments are due to Torben S. Nielsen and Jan K. Møller for producing the point and probabilistic forecasts of wind generation, respectively. Finally, acknowledgments are due to two anonymous reviewers and to an editor for their helpful comments and suggestions.

\section{References}

1. Thor S-E, Weis-Taylor P. Long-term research and development needs for wind energy for the time frame 2000-2020. Wind Energy 2002; 5:73-75.

2. Giebel G, Kariniotakis G, Brownsword R. State of the art on short-term wind power prediction. Technical Report, Anemos Project deliverable report D1.1, 2003 (available online: http://anemos.cma.fr).

3. Pinson P, Kariniotakis G. On-line assessment of prediction risk for wind power production forecasts. Wind Energy 2004; 7:119-132. 
4. Pinson P. Estimation of the uncertainty in wind power forecasting. Ph.D. dissertation, Ecole des Mines de Paris, 2006 (available: www.imm.dtu.dk/ ppp, www.pastel.paristech.org/bib)

5. Nielsen HAa, Nielsen TS, Madsen H, Badger J, Giebel G, Landberg L, Sattler K, Voulund L, Tøfting J. From wind ensembles to probabilistic information about future wind power production - Results from an actual application. Proc. PMAPS Conference, 'Probabilistic Methods Applied to Power Systems', IEEE Conference, Stockholm, Sweden, 2006.

6. Lange M, Focken U. Physical Approach to Short-Term Wind Power Prediction. Springer Verlag: Berlin, 2005.

7. Bremnes JB. A comparison of a few statistical models for making quantile wind power forecasts. Wind Energy 2006; 9:3-11.

8. Gneiting T, Larson K, Westrick K, Genton MG, Aldrich E. Calibrated probabilistic forecasting at the stateline wind energy center - The regime-switching space-time method. Journal of the American Statistical Association 2006; 101:968-979.

9. Juban J, Siebert N, Kariniotakis G. Probabilistic short-term wind power forecasting for the optimal management of wind generation. Proc. PowerTech 2007, IEEE Conference, Lausanne, Switzerland, 2007.

10. Møller JK, Nielsen HAa, Madsen H. Time-adaptive quantile regression. Computational Statistics and Data Analysis 2006; 52:1292-1303.

11. Doherty R, O'Malley M. A new approach to quantify reserve demand in systems with significant installed wind capacity. IEEE Transactions on Power Systems 2005; 20:587-595.

12. Castronuovo ED, Pecas Lopes JA. On the optimization of the daily operation of a wind-hydro power plant. IEEE Transactions on Power Systems 2004; 19:1599-1606.

13. Usaola JG, Angarita JM. Combining hydro-generation and wind energy. Electric Power Systems Research 2007; 77:393-400.

14. Pinson P, Chevallier C, Kariniotakis G. Trading wind generation with short-term probabilistic forecasts of wind power. IEEE Transactions on Power Systems 2007; 22: 1148-1156.

15. Papaefthymiou G. Integration of stochastic generation in power systems. Ph.D. dissertation, Delft University of Technology, Delft, The Netherlands, 2007. ISBN 978-90-8570-186-6, available online: http://www.library.tudelft.nl/ws/search/publications/index.htm

16. Klöckl B. Impacts of energy storage on power systems with stochastic generation. Ph.D. dissertation, Swiss Federal Institute of Technology Zürich (ETH), Zürich, Switzerland, 2007.

17. Papaefthymiou G, Pinson P, Klockl B, Verboomen J. Dynamic sizing of energy storage for hedging wind power forecast uncertainty. working paper, available online: www.imm.dtu.dk/ pp.

18. Pinson P, Nielsen HAa, Møller JK, Madsen H, Kariniotakis G. Nonparametric probabilistic forecasts of wind power: required properties and evaluation. Wind Energy 2007; 10:497-516.

19. Gneiting T, Balabdaoui F, Raftery AE. Probabilistic forecasts, calibration and sharpness. Journal of the Royal Statistical Society B 2007; 69:243-268.

20. Embrecht P, McNeil A, Straumann D. Correlation and dependence in risk management: properties and pitfalls. in Risk Management: Value at Risk and Beyond, Dempster M (ed.), Cambridge University Press: Cambridge, 2002.

21. Madsen H. Time Series Analysis. Chapman \& Hall/CRC: London, 2007.

22. Nielsen TS, Madsen H, Nielsen HAa. Prediction of wind power using time-varying coefficient functions. Proc. IFAC 2002, $15^{\text {th }}$ World Congress on Automatic Control, Barcelona, Spain, 2002.

23. Ahmad IA, Mugdadi AR. Testing normality using kernel methods. Journal of Nonparametric Statistics $2003 ;$ 15:273-288. 